\title{
Vasospasm Post Pituitary Surgery: Systematic Review and 3 Case Presentations
}

\author{
Alireza Mansouri, Aria Fallah, Michael D. Cusimano, Sunit Das
}

\begin{abstract}
Background: Vasospasm is a known complication of aneurysmal subarachnoid hemorrhage and is a major cause of neurological morbidity and mortality. It is infrequently associated with pituitary adenoma surgery. We report three cases and present a systematic review of the literature with a view towards guiding neurosurgeons in the prevention and management of this complication. Results: Including our experience, vasospasm complicating pituitary adenoma surgery has been documented in 29 patients (mean age of 45). All cases occurred in the setting of a postoperative hemorrhage: 21 had a subarachnoid hemorrhage and 10 had a postoperative hematoma requiring evacuation. Initial clinical appearance of delayed cerebral ischemia attributable to vasospasm occurred from postoperative Days 2-13 (most commonly Day 5). Digital subtraction angiography and medical management were the most common diagnostic and therapeutic strategies, respectively. Glasgow Outcome Scores were $\leq 3$ in $59 \%$ of cases. Univariate logistic regression identified later diagnosis of vasospasm and surgery for hematoma evacuation to be independently associated with better outcomes. Conclusion: Vasospasm should be considered in the differential diagnosis of patients demonstrating altered mental or neurological status following pituitary surgery, particularly if there has been postoperative hemorrhage of any degree. Prompt treatment should be instituted to optimize outcome.
\end{abstract}

RÉSUMÉ: Le vasospasme après la chirurgie pituitaire : une revue systématique et présentation de 3 observations. Contexte : Le vasospasme est une complication bien connue de l'hémorragie sous-arachnoïdienne anévrismale et constitue une cause majeure de morbidité neurologique et de mortalité. Il est rarement associé à la chirurgie de l'adénome pituitaire. Nous rapportons 3 observations et nous présentons une revue systématique de la littérature dans l'intention de guider les neurochirurgiens concernant la prévention et le traitement de cette complication. Résultats : Le vasospasme comme complication de la chirurgie pour un adénome pituitaire a été documenté chez 29 patients (âge moyen 45 ans), incluant nos observations cliniques. Toutes ces complications sont survenues dans le cadre d'une hémorragie postopératoire : 21 hémorragies sous-arachnoïdiennes et 10 hématomes postopératoires nécessitant une évacuation de l'hématome. Au point de vue clinique, une ischémie cérébrale retardée attribuable au vasospasme a été observée entre le $2 \mathrm{e}$ jour et le 13e jour après la chirurgie (le plus souvent au $5 \mathrm{e}$ jour). L'angiographie digitale de soustraction et le traitement médical ont été les stratégies diagnostique et thérapeutique les plus fréquemment utilisées. Le score de Galsgow Outcome Scale était de $\leq 3$ chez $59 \%$ des patients. L'analyse de régression logistique univariée a montré qu'un diagnostic subséquent de vasospasme et une chirurgie pour évacuation d'un hématome étaient associés de façon indépendante à de meilleurs résultats. Conclusion : Le vasospasme devrait être inclus dans le diagnostic différentiel de patients dont l'état mental ou l'état neurologique est altéré après la chirurgie pituitaire, particulièrement s'ils ont présenté une hémorragie postopératoire, quelle qu'en soit l'importance. Un traitement rapide devrait être institué afin d'optimiser le résultat.

Can J Neurol Sci. 2012; 39: 767-773

Pituitary adenomas are among the most common primary adult intracranial tumors ${ }^{1}$. Surgical treatment of non-functional adenomas is typically reserved for cases that are symptomatic secondary to mass effect ${ }^{2}$. The surgical approach to these tumors includes the transsphenoidal or transcranial route. Complications commonly associated with pituitary adenoma surgery include diabetes insipidus, cerebral spinal fluid (CSF) leak, transient or permanent visual loss, hypopituitarism, epistaxis, and meningitis ${ }^{3}$. While the risk of hemorrhagic stroke secondary to damage of surrounding vascular structures is well known ${ }^{4}$, the occurrence of vasospasm following pituitary adenoma resection has rarely been documented. We describe three cases of vasospasm following pituitary adenoma surgery from our institution. Further, a summary of cases in the literature is provided with the goal of determining the optimal approach for preventing, diagnosing, and managing this complication.

\section{METHODS}

A systematic search of the literature was performed in November of 2011 using the MEDLINE (1946-2011) and EMBASE (1947-2011) databases to identify relevant cases of vasospasm following pituitary adenoma resection. The following search terms were employed: ['Pituitary' and

From the Division of Neurosurgery (AM, AF, MDC, SD), University of Toronto; Keenan Research Centre (MDC, SD), Li Ka Shing Knowledge Institute, St. Michael's Hospital, Toronto, Ontario, Canada.

Received March 26, 2012. Final Revisions Submitted June 5, 2012. Correspondence to: Alireza Mansouri, St. Michael's Hospital, 30 Bond Street, Toronto, Ontario, M5B 1W8, Canada. Email: alireza.mansouri@utoronto.ca. 
'adenoma'] and ['vasospasm' or 'complication' or 'delayed cerebral ischemia' or 'angioplasty'] and ['surgery' or 'transcranial' or 'transsphenoidal']. This was determined to be the most inclusive strategy while avoiding an overly non-specific search. The search was limited to cases involving human subjects; there were no limitations on language or publication date. Further cases were identified through hand searching. Cases describing a delayed neurological deficit (within the same admission) following any surgical procedure for the resection of a pituitary adenoma were analyzed (including repeat procedures). Only cases with clinical and radiological evidence of vasospasm (either through digital subtraction angiography, computed tomography (CT) perfusion analysis, computed tomography with angiography (CTA) / magnetic resonance angiography (MRA), or transcranial doppler (TCD)) were included in the study. Cases of vasospasm or cerebral ischemia following pituitary apoplexy were not included. No limits were set for the length of follow-up post diagnosis.

The abstracts of the articles identified were independently assessed for appropriateness by two reviewers (AM and AF). Discrepancies in articles identified were resolved through personal discussion. The following variables were extracted for qualitative and quantitative analysis: Date of publication, patient age at time of surgery, gender, tumor type and size, operative approach, initial clinical symptoms (timing of onset of symptoms), method of diagnosis, time to diagnosis (radiological), vessels affected by spasm, treatment strategy, and outcome. For the purpose of statistical analysis the reported outcomes, expressed as a Glasgow Outcome Score (GOS) value, were dichotomized into groups of GOS $\geq 4$ (mild to no deficit) or GOS $\leq 3$ (significant deficit, vegetative state or death).

\section{Statistical analysis}

Univariate regression was performed to identify predictors associated with a dichotomized outcome (GOS 1-3 representing a poor outcome compared to GOS 4-5 representing a good outcome). Independent variables with five or fewer events or those that were biologically implausible to affect outcome were excluded. Any statistically significant predictor of outcome was expressed as an odds ratio with $95 \%$ confidence interval. We also performed multivariable regression, however, the small sample size made robust estimates of parameters for this so unstable that it was abandoned. All statistics were performed on IBM SPSS Statistics 20.

\section{Case 1}

\section{Background}

A 29-year-old right-handed woman presented with a history of progressive headaches, diplopia, visual loss, and amenorrhea. magnetic resonance imaging (MRI) demonstrated a $6.0 \times 5.0$ x6.0 $\mathrm{cm}$ enhancing mass that extended from the sella into the right middle cranial fossa, anterior cranial fossa, and posterior cranial fossa. A two-stage plan was constructed beginning with a subtotal resection through a right fronto-temporal craniotomy for the lateral tumour followed by a purely endoscopic transsphenoidal approach for the midline and sphenoidal lesion. The lateral wall of the cavernous sinus was opened widely and significant amounts of a very vascular tumour were evacuated, contaminating the CSF with blood as well. Pathological analysis revealed a pleomorphic atypical aggressive adenoma producing primarily alpha-subunit and smaller amounts of prolactin and beta-TSH.

\section{Symptom presentation}

Immediate postoperative course was unremarkable. On postoperative Day 5, the patient suddenly developed left-sided hemiplegia and altered level of consciousness. Laboratory studies were normal. Computed tomography scan showed subarachnoid blood, hydrocephalus, and evidence of a right middle carotid artery (MCA) territory infarct. Angiography demonstrated severe diffuse vasospasm involving the supraclinoid segment of the right internal carotid artery (ICA) with narrowing of the right $\mathrm{A} 1$ and no filling of the distal right MCA beyond the M1 segment.

\section{Management}

The patient was treated aggressively with hypervolemia, vasopressor-induced hypertension, and nimodipine, with a gradual improvement of her symptoms. A residual hemiparesis was evident upon discharge which has completely resolved. At 16 years follow-up she has no residual hemiparesis and normal cognitive function $(\mathrm{GOS}=5)$.

\section{CASe 2}

\section{Background}

A 43-year-old right-handed woman presented with progressive diplopia, bitemporal hemianopsia and signs of early Cushing's syndrome. Computed tomography showed a large sellar tumor invading the left cavernous sinus with encasement of the cavernous portion of the left ICA. Portions of the tumor had extended into the prepontine and interpeduncular cisterns. The index operation resulted in a subtotal resection of the tumor which was found to be an aggressive adrenocorticotropic hormone (ACTH) cell adenoma of the Crooke's cell variety. The patient was followed closely with frequent imaging and a second operation was performed six months following the first attempt, which also resulted in a subtotal resection with inadequate decompression of the left optic nerve. The patient then returned to the operating room for a left orbito-fronto-temporal craniotomy for further tumor resection. At surgery, the tumor was found to have invaded the pia of the left optic nerve.

\section{Symptom presentation}

Immediate postoperative course was unremarkable until postoperative day (POD) seven, when she developed headache, vomiting and a mild mixed aphasia. Magnetic resonance imaging showed local edema in and around the frontal and temporal opercula on the left side. Angiogram on POD eight confirmed a mild-to-moderate degree of vasospasm in the left A1 and M1 segments.

\section{Management}

A moderate course of "Triple- $\mathrm{H}$ " therapy (hypertension, hypervolemia, and hemodilution) along with nimodipine was instituted and she demonstrated improvement over the course of the next four days. 
Table 1: Summary of data points obtained through a systematic review of literature on the incidence of vasospasm following pituitary adenoma surgery

\begin{tabular}{|c|c|c|c|c|c|c|c|c|c|c|c|c|}
\hline $\begin{array}{l}1^{\text {st }} \text { author } \\
\text { (year) }\end{array}$ & $\begin{array}{l}\text { Age } \\
(\mathrm{sex})\end{array}$ & Type & Size & Approach & Symptoms & $\begin{array}{l}\text { Onset } \\
\text { (POD) }\end{array}$ & $\begin{array}{l}\text { SAH } \\
(\mathbf{Y} / \mathbf{N})\end{array}$ & $\begin{array}{l}\text { Hematoma* } \\
(\mathbf{Y} / \mathbf{N})\end{array}$ & $\begin{array}{l}\text { Method of } \\
\text { diagnosis }\end{array}$ & $\begin{array}{l}\text { Vessels } \\
\text { affected }\end{array}$ & Treatment & $\begin{array}{l}\text { Outcome } \\
\text { (GOS) }\end{array}$ \\
\hline $\begin{array}{l}\text { Krayenbuhl } \\
(1960)^{* *}\end{array}$ & N/A (F) & $\mathrm{NF}$ & $\mathrm{N} / \mathrm{A}$ & $\begin{array}{l}\text { Subfrontal } \\
\text { TC }\end{array}$ & L hemi- paresis & 4 & $\mathrm{~N} / \mathrm{A}$ & $\mathrm{N}$ & Angiogram & N/A & Papaverine & Resolution (5) \\
\hline \multirow{2}{*}{$\begin{array}{l}\text { Tsunoda } \\
\text { (1972)**** }\end{array}$} & $16(\mathrm{~F})$ & $\mathrm{NF}$ & Suprasellar & TC & Hemi- plegia & 4 & N/A & $\mathrm{N} / \mathrm{A}$ & $\mathrm{N} / \mathrm{A}$ & N/A & $\mathrm{N} / \mathrm{A}$ & $\mathrm{N} / \mathrm{A}$ \\
\hline & $50(\mathrm{~F})$ & $\mathrm{NF}$ & Suprasellar & $\mathrm{TC}$ & Hemi-paresis & N/A & N/A & N/A & $\mathrm{N} / \mathrm{A}$ & N/A & $\mathrm{N} / \mathrm{A}$ & N/A \\
\hline $\begin{array}{l}\text { Takao } \\
(1979)^{* * * *}\end{array}$ & 46 (M) & $\mathrm{NF}$ & Suprasellar & TC & Hemi- paresis & 5 & $\mathrm{~N} / \mathrm{A}$ & $\mathrm{N} / \mathrm{A}$ & $\mathrm{N} / \mathrm{A}$ & N/A & $\mathrm{N} / \mathrm{A}$ & N/A \\
\hline \multirow[t]{3}{*}{$\begin{array}{l}\text { Mawk } \\
\text { (1979) }\end{array}$} & $15(\mathrm{~F})$ & $\mathrm{NF}$ & $\begin{array}{l}\text { Extension } \\
\text { into SOF, } \\
\text { Hypothalamus, } \\
\text { Clivus } \\
\end{array}$ & $\begin{array}{l}\text { R Frontal TC } \\
\text { GTR }\end{array}$ & $\begin{array}{l}\text { Awoke with dense L } \\
\text { hemi- paresis } \\
\text { Lethargy POD4 }\end{array}$ & 6 & $\mathrm{~N} / \mathrm{A}$ & $\mathrm{N}$ & Angiogram & R ICA & Conservative & $\begin{array}{l}\text { Mild affectual } \\
\text { disturbance (5) }\end{array}$ \\
\hline & $42(\mathrm{M})$ & $\mathrm{NF}$ & Suprasellar & $\begin{array}{l}\text { R Frontal TC } \\
\text { GTR }\end{array}$ & $\begin{array}{l}\text { Confusion POD3 } \\
\text { R hemi- paresis }+ \\
\text { obtunded POD5 }\end{array}$ & 3 & $\mathrm{~N} / \mathrm{A}$ & $\mathrm{Y}$ & \begin{tabular}{|l|} 
Angiogram \\
\end{tabular} & L MCA/ACA & Dex & Death (1) \\
\hline & $23(\mathrm{M})$ & $\mathrm{NF}$ & N/A & $\begin{array}{l}\text { R Frontal } \\
\text { TC } \\
\text { STR }\end{array}$ & Dense L hemi- plegia & 10 & $\mathrm{~N} / \mathrm{A}$ & POD8 & Angiogram & R MCA/ACA & Dex & Resolution (5) \\
\hline $\begin{array}{l}\text { Mawk } \\
(1980)\end{array}$ & $22(\mathrm{M})$ & $\mathrm{NF}$ & $\begin{array}{l}5 \times 5 \times 6 \mathrm{~cm} \\
\text { Extension into R } \\
\text { cavernous sinus } \\
\text { and clivus }\end{array}$ & $\begin{array}{l}\text { L Subfrontal } \\
\text { STR }\end{array}$ & L hemiparesis & 3 & $\mathrm{~N}$ & $\mathrm{~N}$ & \begin{tabular}{|l|} 
Angiogram \\
\end{tabular} & R ICA & $\begin{array}{l}\text { Hypervolemic } \\
\text { Therapy }\end{array}$ & Death (1) \\
\hline $\begin{array}{l}\text { Camp } \\
\text { (1980) }\end{array}$ & $33(\mathrm{~F})$ & $\mathrm{NF}$ & Large sellar & $\begin{array}{l}\text { TS } \\
\text { (repeat TS for CSF } \\
\text { leak 1 week later) }\end{array}$ & $\begin{array}{l}\text { Orientation + L hemi- } \\
\text { paresis } \\
\mathrm{L} \mathrm{CN} \mathrm{VI/} \mathrm{VII} \mathrm{palsy} \\
\text { L decerebrate posturing }\end{array}$ & 6 & $\mathrm{~N}$ & $\mathrm{~N}$ & Angiogram & $\begin{array}{l}\text { Diffusely in } \\
\text { proximal } \\
\text { portion of } \\
\text { vessels }\end{array}$ & $\begin{array}{l}\mathrm{HHH}+ \\
\text { Antibiotics }\end{array}$ & Death (1) \\
\hline $\begin{array}{l}\text { Ono } \\
(1981)^{* * * * *}\end{array}$ & $48(\mathrm{M})$ & $\mathrm{NF}$ & Suprasellar & TC & Lethargy + hemi- paresis & 7 & N/A & $\mathrm{N} / \mathrm{A}$ & $\mathrm{N} / \mathrm{A}$ & $\mathrm{N} / \mathrm{A}$ & $\mathrm{N} / \mathrm{A}$ & $\mathrm{N} / \mathrm{A}$ \\
\hline $\begin{array}{l}\text { Hyde-Rowan } \\
\text { (1983) }\end{array}$ & $30(\mathrm{~F})$ & $\begin{array}{l}\text { GH + } \\
\text { PRL }\end{array}$ & $\mathrm{N} / \mathrm{A}$ & $\begin{array}{l}\text { TS } \\
1400 \mathrm{ml} \text { blood loss } \\
\text { intra-op }\end{array}$ & $\begin{array}{l}\text { MI } \\
\text { Comatose + decorticate } \\
\text { posturing: awoke POD5 } \\
\text { Apnea + aphasia + } \\
\text { obtunded POD6 }\end{array}$ & 2 & $\begin{array}{l}\text { Y } \\
\text { POD2 } \\
\text { (LP) }\end{array}$ & $\mathrm{N}$ & \begin{tabular}{|l|} 
Angiogram \\
\end{tabular} & $\begin{array}{l}\mathrm{B} / \mathrm{L} \\
\text { supraclinoid } \\
\text { ICAs }\end{array}$ & Conservative & Death (1) \\
\hline $\begin{array}{l}\text { Barrow } \\
\text { (1990) }\end{array}$ & $50(\mathrm{M})$ & PRL & $\begin{array}{l}\text { Suprasellar } \\
\text { L frontal lobe }\end{array}$ & $\begin{array}{l}\text { L Frontal } \\
\text { TC } \\
\text { STR } \\
\text { (Second stage TS } \\
\text { STR) } \\
\end{array}$ & $\begin{array}{l}R \text { hemi- paresis }+R \text { sided } \\
\text { focal seizure }+L \text { eye } \\
\text { blindness }+ \text { dysphasia }\end{array}$ & $\begin{array}{l}7 \\
\text { (POD 2 } \\
\text { after } \\
\text { second } \\
\text { stage TS) }\end{array}$ & $\mathrm{N}$ & $\mathrm{N}$ & \begin{tabular}{|l}
$\begin{array}{l}\text { Angiogram } \\
\text { (POD9) }\end{array}$ \\
\end{tabular} & $\begin{array}{l}\text { L ACA + ICA } \\
\text { (supra- clinoid) }\end{array}$ & $\mathrm{HHH}$ & $\begin{array}{l}\text { Gradually } \\
\text { better } \\
\text { L eye blind (3) }\end{array}$ \\
\hline $\begin{array}{l}\text { Aoki } \\
\text { (1995) }\end{array}$ & $51(\mathrm{~F})$ & $\mathrm{NF}$ & $\begin{array}{l}\text { Suparsellar } \\
\text { L cavernous sinus }\end{array}$ & $\begin{array}{l}\text { L Cranio- orbito- } \\
\text { zygomatic } \\
\text { STR }\end{array}$ & "Clinical deterioration" & 5 & N/A & N/A & CTA & B/L ICAs & $\mathrm{HHH}$ & Resolution (5) \\
\hline \multirow[t]{2}{*}{$\begin{array}{l}\text { Cervoni } \\
\text { (1996) }\end{array}$} & $51(\mathrm{M})$ & $\mathrm{NF}$ & Suprasellar & TS & $\begin{array}{l}\text { Orientation + L hemi- } \\
\text { paresis }\end{array}$ & 4 & $\begin{array}{l}\mathrm{Y} \\
\mathrm{POD} 4 \\
\end{array}$ & $\mathrm{~N} / \mathrm{A}$ & TCD & R MCA/ACA & $\begin{array}{l}\mathrm{HHH}+ \\
\text { nimodipine }\end{array}$ & Resolution (5) \\
\hline & $48(\mathrm{~F})$ & $\mathrm{NF}$ & Suprasellar & $\begin{array}{l}\text { R Fronto- temporal } \\
\text { TC }\end{array}$ & $\begin{array}{l}\text { Progressive L hemi- } \\
\text { paresis }\end{array}$ & 5 & $\begin{array}{l}\text { Y } \\
\text { POD5 }\end{array}$ & $\mathrm{N} / \mathrm{A}$ & TCD & R MCA & $\begin{array}{l}\mathrm{HHH}+ \\
\text { nimodipine }\end{array}$ & Death from MI (1) \\
\hline $\begin{array}{l}\text { Friedman } \\
(2001)\end{array}$ & $41(\mathrm{M})$ & GH & N/A & $\begin{array}{l}\text { TS } \\
\text { GTR }\end{array}$ & $\begin{array}{l}\text { - Drowsy POD10 } \\
\text { - Mild aphasia } \\
\text { - R arm weaknessPOD11 }\end{array}$ & 10 & $\mathrm{~N}$ & $\mathrm{~N}$ & Angiogram & $\begin{array}{l}\text { Severe L } \\
\text { supraclinoid } \\
\text { ICA }\end{array}$ & Angioplasty & Resolution (5) \\
\hline $\begin{array}{l}\text { Nishioka } \\
\text { (2001) }\end{array}$ & $41(\mathrm{M})$ & FSH & $\mathrm{N} / \mathrm{A}$ & TS & $\begin{array}{l}\text { Orientation } \\
\text { L hemi- paresis POD12 } \\
\text { Aphasia + R hemi- paresis } \\
\text { POD } 17\end{array}$ & 12 & $\begin{array}{l}\text { Y } \\
\text { POD0 }\end{array}$ & $\mathrm{N}$ & Angiogram & $\begin{array}{l}\mathrm{R} \mathrm{MCA} / \mathrm{ACA} / \\
\text { diatal ICA }\end{array}$ & $\begin{array}{l}\text { Papaverine } \\
\text { POD1 } \\
\text { HHH } 4 \text { weeks } \\
\text { later }\end{array}$ & $\begin{array}{l}\text { Hypo- pituitarism } \\
\text { (5) }\end{array}$ \\
\hline $\begin{array}{l}\text { Kasliwal } \\
\text { (2008) }\end{array}$ & $34(\mathrm{~F})$ & $\mathrm{NF}$ & Suprasellar & $\begin{array}{l}\text { TS } \\
\text { STR }\end{array}$ & $\begin{array}{l}\text { R sided hemi-paresis, } \\
\text { focal convulsions }\end{array}$ & 13 & $\mathrm{~N}$ & $\mathrm{Y}$ & $\begin{array}{l}\text { Angiogram + } \\
\text { TCD }\end{array}$ & $\begin{array}{l}\text { L ICA } \\
\text { bifurcation }\end{array}$ & $\begin{array}{l}\text { Hypervolemic } \\
\text { Therapy }\end{array}$ & Death (1) \\
\hline \multirow[t]{3}{*}{$\begin{array}{l}\text { Puri **** } \\
\text { (2010) }\end{array}$} & $\mathrm{N} / \mathrm{A}$ & $\mathrm{NF}$ & N/A & TS & Global aphasia & 5 & $\mathrm{Y}$ & $\mathrm{Y}$ & Angiogram & $\begin{array}{l}\text { Diffuse (worse } \\
\text { in ACAs) }\end{array}$ & Verapamil & $\begin{array}{l}\text { B/L L/E weakness } \\
\text { (3) }\end{array}$ \\
\hline & $\mathrm{N} / \mathrm{A}$ & $\mathrm{NF}$ & $\mathrm{N} / \mathrm{A}$ & TS & Behavioral changes & 9 & $\mathrm{Y}$ & $\mathrm{Y}$ & $\begin{array}{l}\text { Angiogram }+ \\
\text { CTA } \\
\end{array}$ & Diffuse & Verapamil & "Lasting deficit" (3) \\
\hline & $\mathrm{N} / \mathrm{A}$ & $\mathrm{NF}$ & $\mathrm{N} / \mathrm{A}$ & TS & Depressed mental status & 5 & $\mathrm{Y}$ & $\mathrm{Y}$ & $\begin{array}{l}\text { Angiogram }+ \\
\text { CTA }\end{array}$ & Diffuse & Verapamil & Death (1) \\
\hline \multirow[t]{2}{*}{$\begin{array}{l}\text { Popugaev } \\
\text { (2011) }\end{array}$} & $45(\mathrm{M})$ & $\mathrm{NF}$ & Suprasellar & TS & Meningismus & 4 & $\begin{array}{l}\mathrm{Y} \\
\mathrm{POD} 4\end{array}$ & $\mathrm{~N}$ & \begin{tabular}{|l} 
TCD (POD4) \\
Angiogram \\
(POD23) \\
\end{tabular} & $\begin{array}{l}\mathrm{B} / \mathrm{L} \text { ICA }+ \\
\mathrm{MCA}\end{array}$ & $\begin{array}{l}\text { IV Antibiotics } \\
\text { started POD7 }\end{array}$ & GOS 4 \\
\hline & $52(\mathrm{~F})$ & $\mathrm{NF}$ & Suprasellar & $\begin{array}{l}\text { TS } \\
\text { STR } \\
\end{array}$ & $\begin{array}{l}\text { Delirium } \\
\text { Respiratory insufficiency }\end{array}$ & 4 & $\mathrm{~N} / \mathrm{A}$ & $\mathrm{N} / \mathrm{A}$ & TCD & L MCA & $\begin{array}{l}\text { IV Antibiotics } \\
\text { started POD4 }\end{array}$ & GOS 5 \\
\hline \multirow[t]{3}{*}{$\begin{array}{l}\text { Zada } \\
\text { (2011) }\end{array}$} & $59(\mathrm{M})$ & $\mathrm{NF}$ & $\begin{array}{l}4 \times 2.9 \mathrm{~cm} \\
\text { Suprasellar } \\
\text { Floor of third } \\
\text { Encasing ACAs }\end{array}$ & $\begin{array}{l}\text { TS } \\
\text { STR }\end{array}$ & $\begin{array}{l}\text { Drowsy }+ \text { R pupil dilation } \\
\text { POD2 } \\
\text { Global aphasia }+ \text { B/L leg } \\
\text { weakness POD5 }\end{array}$ & 2 & $\begin{array}{l}\mathrm{Y} \\
\text { POD2 }\end{array}$ & \begin{tabular}{|l|l} 
Y \\
POD2
\end{tabular} & $\begin{array}{l}\text { Angiogram + } \\
\text { CTA }\end{array}$ & $\begin{array}{l}\text { Diffuse } \\
\text { (ACAs severe) }\end{array}$ & Verapamil x 3 & $\begin{array}{l}\text { Short- term memory } \\
\text { and VF deficit (4) }\end{array}$ \\
\hline & 66 (M) & $\mathrm{NF}$ & $\begin{array}{l}4.9 \times 3.6 \mathrm{~cm} \\
\text { Suprasellar } \\
\text { Frontal lobe }\end{array}$ & $\begin{array}{l}\text { TS } \\
\text { STR }\end{array}$ & $\begin{array}{l}\text { Decreased LOC } \\
\text { R pupil dilation POD5 } \\
\text { Obtunded POD8 }\end{array}$ & 8 & $\mathrm{~N}$ & $\begin{array}{l}\text { Y } \\
\text { POD5 }\end{array}$ & $\begin{array}{l}\text { Angiogram }+ \\
\text { MRA }\end{array}$ & $\begin{array}{l}\text { Severe B/L } \\
\text { ACA + L PCA }\end{array}$ & Verapamil & Death (1) \\
\hline & $36(\mathrm{~F})$ & FSH & $\begin{array}{l}2.9 \times 2.1 \mathrm{~cm} \\
\text { Suprasellar }\end{array}$ & $\begin{array}{l}\text { TS- GTR (Previous } \\
\text { STR via TS) }\end{array}$ & $\begin{array}{l}\text { Agitation } \\
\text { Expressive aphasia }\end{array}$ & 9 & \begin{tabular}{|l|} 
Y \\
POD 0
\end{tabular} & $\mathrm{~N}$ & CTA & $\begin{array}{l}\text { Diffuse (infarct } \\
\text { in B/L ACA } \\
\text { territories) }\end{array}$ & $\begin{array}{l}\text { HHH + } \\
\text { verapamil x } 4\end{array}$ & $\begin{array}{l}\text { Stable pan- hypo- } \\
\text { pituitarism (5) }\end{array}$ \\
\hline \multirow[t]{3}{*}{$\begin{array}{l}\text { Toronto } \\
\text { (2011) }\end{array}$} & $29(\mathrm{~F})$ & $\begin{array}{l}\text { GH + } \\
\text { PRL }\end{array}$ & $\begin{array}{l}6 \times 5 \times 6 \mathrm{~cm} \\
\mathrm{R} \text { mid skull base } \\
\text { Ant.\& post. } \\
\text { fossa }\end{array}$ & $\begin{array}{l}\text { Fronto- temporal } \\
\text { TC }\end{array}$ & L sided hemi- plegia & 5 & $\mathrm{Y}$ & $\mathrm{N}$ & \begin{tabular}{|l|} 
Angiogram \\
\end{tabular} & $\begin{array}{l}\text { R ICA/MCA/ } \\
\text { ACA }\end{array}$ & $\begin{array}{l}\mathrm{HHH}+ \\
\text { nimodipine }\end{array}$ & Resolution (5) \\
\hline & $45(\mathrm{~F})$ & ACTH & $\begin{array}{l}\text { Cavernous } \\
\text { sinuses } \\
\text { Middle cranial } \\
\text { fossa }\end{array}$ & $\begin{array}{l}\text { Orbito- fronto- } \\
\text { temporal (3 } \\
\text { previous TS } \\
\text { surgeries) } \\
\text { All = STR } \\
\end{array}$ & Dysphasia & 7 & $\mathrm{Y}$ & $\mathrm{Y}$ & \begin{tabular}{|l|} 
Angiogram \\
(POD8)
\end{tabular} & $\mathrm{L}$ Al/M1 & $\begin{array}{l}\begin{array}{l}\text { HHH + } \\
\text { nimodipine }\end{array} \\
\end{array}$ & $\begin{array}{l}\text { Mild speech deficits } \\
\text { (4) }\end{array}$ \\
\hline & 75 (M) & $\mathrm{NF}$ & $\begin{array}{l}2 \times 1.7 \times 2.8 \mathrm{~cm} \\
\text { Floor of } 3^{\text {rd }}\end{array}$ & TS & $\begin{array}{l}\text { Decreased level of } \\
\text { consciousness }+\mathrm{R} \text { sided } \\
\text { hemi- paresis }\end{array}$ & 7 & $\mathrm{Y}$ & $\mathrm{Y}$ & CT perfusion & L ICA/MCA & Permissive HTN & $\begin{array}{l}\text { Difficulty } \\
\text { ambulating (3) }\end{array}$ \\
\hline
\end{tabular}

\$ Abbreviations: GH, Growth Hormone; FSH, Follicle Stimulating Hormone; PRL, Prolactine; ACTH, Adrenocorticotrophic Hormone; NF, NonFunctional; TS, Transsphenoidal; TC, Transcranial; STR, Sub Total Resection; GTR, Gross Total Resection; POD, Postoperative Day; SAH, Subarachnoid Hemorrhage; CTA, Computed Tomography with Angiography; MRA, Magnetic Resonance Angiography; TCD, Transcranial Doppler; ICA, Internal Carotid Artery; MCA, Middle Cerebral Artery; ACA, Anterior Cerebral Artery; PCA, Posterior Cerebral Artery; B/L, Bilateral; L/E, Lower extremity; HHH, Hypertension-Hypervolemia-Hemodilution therapy; HTN, Hypertension; GOS, Glasgow Outcome Scale.

* Indicates hematoma significant enough to require evacuation; ** Indicates article in German; *** Indicates publication type as abstract only;

**** Indicates articles in Japanese 


\section{Outcome}

Following discharge, the patient underwent adjuvant therapy with radiation and temozolomide. However, the tumor recurred once again and a further transsphenoidal approach was necessary. A near-total resection of the tumor was obtained. On most recent follow-up the patient demonstrated mild deficits in medial and lateral movements of the left eye, intermittent headaches, and mild speech difficulties $(\mathrm{GOS}=4)$.

\section{CASE 3}

\section{Background}

A 75-year-old right-handed man presented with a one year history of decreased peripheral vision, visual blurring, and a three to four week history of new-onset headaches. Magnetic resonance imaging showed a pituitary macroadenoma measuring $2.0 \times 1.7 \times 2.8 \mathrm{~cm}$ extending superiorly into the suprasellar cisten with mass effect on the chiasm and floor of the third ventricle. Displacement of cavernous ICAs with bilateral invasion of the cavernous sinuses was also noted. An endoscopic extended transsphenoidal resection of the tumor was undertaken. The optic chiasm and the right optic nerve were decompressed. Significant amount of venous bleeding from the left cavernous sinus was noted but hemostasis was obtained with gel foam and irrigation. A small perforation was inadvertently created in the arachnoid veil resulting in an immediate CSF leak which was managed intraoperatively.

\section{Symptom presentation}

In the immediate postoperative period the patient was cogent and reported an improvement in his visual acuity. Twenty four hours after surgery the patient developed a decrease in the level of consciousness. Computed tomography showed acute hemorrhage in the suprasellar space, just anterior to the genu of the corpus callosum, along with the interpeduncular fossa, the right pre-pontine cistern, and in the third and lateral ventricles. Hydrocephalus was also noted. The CTA did not reveal evidence of vascular injury or vasospasm.

The patient was taken back to the operating room for endoscopic transsphenoidal evacuation of the suprasellar hematoma. An external ventricular drain was inserted at the same setting. The patient gradually improved until POD seven when he once again became somnolent and developed a rightsided hemiplegia. The CT perfusion imaging demonstrated a significant narrowing of left supraclinoid ICA with focal narrowing extending to level of the left MCA resulting in hypoperfusion of the left hemisphere.

\section{Management}

The patient was managed via permissive hypertension and hypervolemia. A CT scan on POD ten showed evidence of infarcts in the left globus pallidus and genu of internal capsule. These infarcts were confirmed by MRI three weeks later.

\section{Outcome}

Over the course of the next month he demonstrated gradual improvement, but remained significantly impaired. While able to follow simple commands, he required assistance with
Table 2: Demographics and pre/post-operative tumor information

\begin{tabular}{c|c}
\hline & Frequency (Percentage) \\
\hline Male & $13(50 \%)$ \\
\hline Female & $13(50 \%)$ \\
\hline Not specified & 3 \\
\hline $1960-1969$ & $1(3 \%)$ \\
\hline $1970-1979$ & $6(21 \%)$ \\
\hline $1980-1989$ & $4(14 \%)$ \\
\hline $1990-1999$ & $4(14 \%)$ \\
\hline $2000-2011$ & $14(48 \%)$ \\
\hline Type of tumor & $22(76 \%)$ \\
\hline Non-functional & $7(24 \%)$ \\
\hline Functional & $15(52 \%)$ \\
\hline Surgical approach & $14(48 \%)$ \\
\hline Transsphenoidal & \\
\hline Transcranial & $12(60 \%)^{*}$ \\
\hline Operative outcome & $8(40 \%)^{*}$ \\
\hline Sub-total resection & $9(\mathrm{~N} / \mathrm{A})$ \\
\hline Gross-total resection & \\
\hline Not specified & \\
\hline
\end{tabular}

* Indicates percentage of those reported

ambulation. At three month follow-up, he was found to have a mild residual hemiparesis, but displayed severe abulia $(\mathrm{GOS}=3)$.

\section{RESULTS}

The initial search yielded 844 articles; after review of abstracts for appropriateness, 20 cases (12 articles) were identified as relevant. Six additional cases (five articles) were identified by searching through the references of the above articles. Including the three cases from our institution, there have been a total of 29 cases of vasospasm following pituitary adenoma resection reported in the literature (Table 1) ${ }^{5-21}$. Patient demographics, preoperative tumor information, along with operative approach and outcome have been summarized in Table 2. Nearly half of the cases were reported within the past decade. Patient age at presentation ranged from 15 to 75 with a mean age of 45 at time of surgery. Gender, operative approach, or extent of surgical resection were not predictive of the risk of vasospasm. Initial signs and symptoms of deterioration such as changes in mental status $(56 \%)$, motor deficits $(62 \%)$, and speech difficulties (28\%) were detected on POD 2-13; the majority of cases were detected on POD 5. Among reports commenting on the presence of subarachnoid hemorrhage (SAH), 70\% (14/20) of vasospasm cases were associated with SAH while $34 \%$ had developed postoperative hemorrhage significant enough to require surgical evacuation. A GOS score of $\leq 3$ was observed in half of the patients with evidence of SAH.

A variety of diagnostic and treatment modalities were utilized (Table 3). Angiography was used to diagnose vasospasm in $77 \%$ of cases (used as the only tool in 54\% and as an adjunct in $23 \%$ of cases). The majority of cases (68\%) employed medical measures such as "Triple H", nimodipine, intra-arterial 
verapamil, and IV papaverine. Conservative measures (28\% of cases) included the use of antibiotics for suspected meningitis, dexamethasone, permissive hypertension or clinical monitoring. Only one case reported the use of angioplasty as a treatment strategy and with good success $(\mathrm{GOS} \geq 4)$. More than half of the cases overall were associated with a GOS score of $\leq 3(60 \%)$; eight cases $(32 \%)$ ultimately resulted in patient death. Subanalysis in this group did not suggest a definitive association of mortality with the presence of SAH or extensive hematoma.

On univariate analysis a trend was observed for patients with "Hematoma requiring evacuation and a good outcome with an OR of 5.5 ( $\mathrm{p}=0.08)$. We were unable to perform multivariate analysis to identify independent predictors of outcome due to the low sample size.

\section{Discussion}

The incidence of vasospasm following $\mathrm{SAH}$ is welldescribed $^{22,23}$. While SAH-related vasospasm is most commonly associated with rupture of an intracranial aneurysm, the literature contains reports of cases associated with surgical resection of skull base tumors as well ${ }^{7,12,14,24,25}$. Further, there have been a limited number of published cases of vasospasm following pituitary surgery. In this study, we have reported findings from three cases at our institution and have systematically reviewed the literature and identified an additional 26 cases. Through this approach we have been able to gain further insight into the phenomenon of vasospasm following pituitary surgery.

\section{Clinical presentation}

The presenting symptoms noted in our case series were generally reflective of those commonly encountered in patients developing vasospasm. However, the differential diagnosis for such presenting symptoms in the postoperative period following pituitary surgery is broad and includes multiple other etiologies: metabolic (hyponatremia, hypernatremia, hypocortisolemia), systemic (hypotension, hypoxia, hypercarbia), infectious (meningitis, sepsis), and neurologic (stroke, hydrocephalus, mass effect from hemorrhage or edema, seizure $)^{26}$. Given that these diagnoses are more common following pituitary adenoma surgery emphasizes the importance of maintaining a high index of suspicion for vasospasm, particularly following anatomically challenging cases or those associated with hemorrhage.

\section{Putative mechanisms}

Clinical vasospasm associated with aneurysmal rupture portends a poor prognosis, with reported death in $7 \%$ of cases and a severe, lasting deficit in an additional $7 \%^{27}$. The overall rate of severe deficit and mortality in our series was substantially higher, at $59 \%$ and $28 \%$, respectively. This is attributable to our data including extreme cases and the probability of mild cases being undiagnosed is high. Aside from SAH, other factors relevant to pituitary surgery that can contribute to the development of vasospasm include mechanical intraoperative manipulation of cerebral arteries ${ }^{28}$, meningitis ${ }^{11}$, irritation of vessels by packing material ${ }^{13}$ or the release of vasoactive peptides and hormones from the hypothalamus following surgery within the sellar/suprasellar region ${ }^{29}$. Anatomy may also play a role. Hemorrhage from pituitary surgery is also most
Table 3: Summary of diagnostic methods, management approaches, and outcomes

\begin{tabular}{c|c}
\hline & Frequency (Percentage) \\
\hline Diagnostic modality & $14(54 \%)$ \\
\hline Angiography & $3(11.5 \%)$ \\
\hline CT perfusion/ CTA/MRA & $3(11.5 \%)$ \\
\hline Trans-cranial Doppler & $6(23 \%) \dagger$ \\
\hline Angiography + other diagnostic modality & 3 \\
\hline Not specified & $1(4 \%)$ \\
\hline Treatment approach & $17(68 \%)$ \\
\hline Endovascular & $7(28 \%)$ \\
\hline Medical & 4 \\
\hline Conservative & $10(40 \%)$ \\
\hline Not specified & $15(60 \%)$ \\
\hline Gutcome $\quad$ GOS & 4 \\
\hline GOS $\leq 3$ &
\end{tabular}

$\dagger 4$ involved the use of CTA/MRA while 2 involved the use of TCD

likely to maximally affect the basal cisterns of the entire Circle of Willis. Thus the likelihood of diffuse vasospasm involving anterior and middle cerebral arteries bilaterally would be higher than a localized SAH, say around a single middle cerebral artery.

\section{Diagnostic modalities}

In our series, conventional angiography was the primary diagnostic tool, followed by CTA, TCD, MRA, and CT perfusion studies. Angiography has been established as the gold standard for the diagnosis of vasospasm; however, CTA and MRA have a reasonable specificity and sensitivity for detection of vasospasm and can often be sufficient for diagnosis ${ }^{30,31}$. If CTA or MRA are not suggestive of vasospasm but there is radiographic evidence of SAH and clinical suspicion of vasospasm, early angiography should be performed in patients with a delayed neurological decline as a delay in diagnosis may have an effect on outcome.

\section{Therapeutic approaches}

As in vasospasm associated with aneurysmal SAH, a variety of therapeutic strategies can be utilized for the management of vasospasm complicating pituitary surgery. In our series, the majority of cases involved the use of "Triple-H" therapy, typically in conjunction with nimodipine, followed by intraarterial delivery of an antispasmodic such as verapamil or papaverine or mechanical angioplasty if needed. "Triple-H" resulted in a good outcome in 7 of 11 patients. In the remaining four cases, however, mortality was the outcome; one of these was from a myocardial infarct. In certain cases where meningitis was presumed to be the underlying cause of vasospasm, appropriate antibiotic therapy resulted in a good outcome. In other cases, however, conservative management had a higher association with poor outcome. Mechanical angioplasty was performed in one case with a resultant good outcome. While our study was under-powered for the identification of the superiority of one management approach over another, the prompt 
institution of appropriate therapy for vasospasm in a monitored neurosurgical ICU setting, with consideration of patient comorbidities, seems prudent.

In our analysis, an unexpected trend was observed correlating surgical evacuation of hematoma within the tumor bed and an overall good patient outcome. It is possible that this was a spurious finding that occurred by chance as evidenced by the wide confidence interval. However, it is possible that early relief of mass effect can result in better outcomes; the size of the hematoma was often not reported. In addition, other unknown underlying mechanisms could be contributing to this finding, and may be independent of any effect on vasospasm. Regardless of mechanism, early evacuation of a hematoma in a patient with an altered level of consciousness is a variable that should be considered and further analyzed in future studies.

\section{Limitations}

Although our search of the literature was able to identify several cases, the small number of cases identified was not sufficient to achieve adequate statistical power to identify clinically important associations. It is likely that the incidence of vasospasm following pituitary surgery is higher than we have indicated, and that only the most severe cases have been reported in the literature (i.e. publication bias). Furthermore, our data points were extracted from case reports and case series. Therefore, our study is limited by a high level of reporting bias. Among the cases identified, incomplete reporting of data such as presence and amount of SAH, degree of spasm, outcomes in terms of GOS, and follow-up further complicated our study. This limitation was particularly important for GOS, where inferences were made based on the reported descriptors of patient outcome. In addition, specific information regarding the treatment center is also missing; institutions with a dedicated neurosurgical ICU with the expertise to manage aneurysmal $\mathrm{SAH}$ and vasospasm are likely to have better outcomes.

Overall, this is the first attempt in the neurosurgical literature to summarize the reported cases of vasospasm following pituitary adenoma surgery. Our study enabled the observation of trends and patterns in this patient population and served as a hypothesis-generating exercise for further studies. Future directions would include the accumulation of additional studies to garner higher statistical power. Also, while there are theories regarding the underlying pathophysiology of vasospasm following pituitary adenoma surgery, further research is necessary for better characterization.

\section{CONCLUSION}

Through this systematic review, we have been able to gain further insight into the phenomenon of vasospasm following pituitary surgery. Given the relatively high morbidity and mortality rates observed in this series, a high level of vigilance for vasospasm is necessary in patients undergoing an extensive resection of a pituitary adenoma. Although the differential diagnosis for an altered level of consciousness or deficits in motor functions and speech is broad, vasospasm is a rare but important diagnosis to consider during the immediate postoperative period. The astute clinician will consider the diagnosis in the setting of pituitary surgery complicated by hemorrhage and remain vigilant and institute preventive measures to avoid potentially fatal or disabling complications seen in these patients.

\section{REFERENCES}

1. Abbott J, Kirkby GR. Acute visual loss and pituitary apoplexy after surgery. BMJ. 2004 Jul 24;329(7459):218-19.

2. Jane JA, Jr., Laws ER, Jr. Surgical management of pituitary adenomas. Singapore Med J. 2002 Jun;43(6):318-23.

3. Barzaghi LR, Losa M, Giovanelli M, Mortini P. Complications of transsphenoidal surgery in patients with pituitary adenoma: experience at a single centre. Acta Neurochirurgica. 2007;149 (9):877-85; discussion 85-6.

4. Raymond J, Hardy J, Czepko R, Roy D. Arterial injuries in transsphenoidal surgery for pituitary adenoma; the role of angiography and endovascular treatment. AJNR Am J Neuroradiol. 1997 Apr;18 (4):655-65.

5. Friedman JA, Meyer FB, Wetjen NM, Nichols DA. Balloon angioplasty to treat vasospasm after transsphenoidal surgery. Case illustration. J Neurosurg. 2001 Aug;95(2):353.

6. Nishioka $\mathrm{H}$, Ito $\mathrm{H}$, Haraoka J. Cerebral vasospasm following transsphenoidal removal of a pituitary adenoma. Br J Neurosurg. $2001 \mathrm{Feb} ; 15(1): 44-7$.

7. Zada G, Du R, Laws ER, Jr. Defining the "edge of the envelope": patient selection in treating complex sellar-based neoplasms via transsphenoidal versus open craniotomy. J Neurosurg. $2011 \mathrm{Feb}$; 114(2):286-300.

8. Mawk JR, Ausman JI, Erickson DL, Maxwell RE. Vasospasm following transcranial removal of large pituitary adenomas. Report of three cases. J Neurosurg. 1979 Feb;50(2):229-32.

9. Krayenbuhl H. Beitrag zur Frage des cerebralen angiospastischen Insultes. Schweiz Med Wochenschr. 1960;90:961-5.

10. Hyde-Rowan MD, Roessmann U, Brodkey JS. Vasospasm following transsphenoidal tumor removal associated with the arterial changes of oral contraception. Surg Neurol. 1983 Aug;20 (2):120-4

11. Popugaev KA, Savin IA, Lubnin AU, et al. Unusual cause of cerebral vasospasm after pituitary surgery. Neurol Sci. 2011 Aug;32(4):673-80.

12. Cervoni L, Salvati M, Santoro A. Vasospasm following tumor removal: report of 5 cases. Ital J Neurol Sci. 1996 Aug;17(4): 291-4.

13. Camp PE, Paxton HD, Buchan GC, Gahbauer H. Vasospasm after trans-sphenoidal hypophysectomy. Neurosurgery. 1980 Oct; 7(4): 382-6.

14. Aoki N, Origitano TC, al-Mefty O. Vasospasm after resection of skull base tumors. Acta Neurochirurgica. 1995;132(1-3):53-8.

15. Barrow DL, Tindall GT. Loss of vision after transsphenoidal surgery. Neurosurgery. 1990 Jul;27(1):60-8.

16. Puri AZ, Zarzour G, Laws EH. Frerichs K. Symptomatic cerebral vasospasm after trans-sphenoidal resection of pituitary macroadenomas. J Neurointerv Surg. 2010;2(S1):A37.

17. Ono MM, Nukui H. Kawafuchi J. Vasospasm following removal of a large pituitary adenoma by the subfrontal approach. Report of a case and review of the literature. Neurologica MedicoChirurgica. 1981;21(6):609-14.

18. Tsunoda MOT, Kuramae H, Takagi H, Nakamura N, Miyasaka Y. Two cases of cerebral angiospasm after removal of pituitary tumor. Discussion of clinical course and pathogenesis. Tokyo: Neuron Co; 1972; 7-17.

19. Takao A WY, Kameda H, Tsunami M, Satoh K, Ueda, H MT. A case complicated with severe vasospasm after total removal of a pituitary tumor. Rinshoshinkei. 1979;19:772.

20. Kasliwal MK, Srivastava R, Sinha S, Kale SS, Sharma BS. Vasospasm after transsphenoidal pituitary surgery: a case report and review of the literature. Neurology India. 2008 Jan-Mar;56 (1):81-3.

21. Mawk JR. Vasospasm after pituitary surgery. J Neurosurg. 1980; 58:972.

22. Kassell NF, Kongable GL, Torner JC, Adams HP, Jr., Mazuz H. Delay in referral of patients with ruptured aneurysms to neurosurgical attention. Stroke. 1985 Jul-Aug;16(4):587-90. 
23. Kassell NF, Torner JC, Haley EC, Jr., Jane JA, Adams HP, Kongable GL. The International Cooperative Study on the Timing of Aneurysm Surgery. Part 1: Overall management results. J Neurosurg. 1990 Jul;73(1):18-36.

24. LeRoux PD, Haglund MM, Mayberg MR, Winn HR. Symptomatic cerebral vasospasm following tumor resection: report of two cases. Surg Neurol. 1991 Jul;36(1):25-31

25. MacDonald RL. Cerebral Vasospasm. San Diego, California: Academic Press; 2001.

26. MacDonald RL. Management of cerebral vasospasm. Neurosurgical Rev. 2006;29:179-93.

27. Sen J, Belli A, Albon H, Morgan L, Petzold A, Kitchen N. Triple-H therapy in the management of aneurysmal subarachnoid haemorrhage. Lancet Neurol. 2003;2(10):614-20.

28. Laws ER. Vascular complications of transsphenoidal surgery. Pituitary. 1999;2(2):163-70.
29. Wilson JL, Field JR. The production of intracranial vascular spasm by hypothalamic extract. J Neurosurg. 1974;40:473-9.

30. Anderson GB, Findlay JM. CT angiography for the detection of cerebral vasospasm in patients with acute subarachnoid hemorrhage. Am J Neuroradiol. 2000;21:1011-15.

31. Tamatani S, Fujii Y, Koike T, Tanaka R. Detection of delayed cerebral vasospasm, after rupture of intracranial aneurysms, by magnetic resonance angiography (comment). Neurosurgery. 1997;40:748-53 